\title{
Chaos and Stochasticity in Deterministically Generated Multifractal Measures*
}

\author{
Nelson Obregón Neira** \\ Carlos E. Puente***
}

Resumen: En este artículo se muestra un procedimiento geométrico determinístico, la metodología fractal-multifractal (FM) [Puente, 1992], dirigido a la representación holística de series de tiempo geofísicas como proyecciones desde funciones de interpolación fractales produciendo un conjunto de $1 / f^{\beta}$ series multifractales que incluyen tanto señales "caóticas" como "estocásticas". Dicho resultado inesperado y aparentemente contradictorio, dado el determinismo intrínseco de la representación, sugiere que el concepto de las proyecciones podría ser útil para estudiar la complejidad de un variado número de eventos naturales. Se presentan los ejemplos de la aproximación FM que evidencian dicho comportamiento junto con un breve repaso de los conceptos básicos del análisis no lineal de series de tiempo.

Abstract: A deterministic geometric procedure, the Fractal-Multifractal (FM) Methodology [Puente, 1992], aimed at representing geophysical time series wholistically as projections of fractal interpolating functions, is shown to produce a variety of $1 / f^{\beta}$ multifractal series that include both "chaotic" and "stochastic" signals. This unexpected and apparently contradictory result, given the intrinsic determinism of the representation, suggests that the concept of projections may be useful to study the complexity of a variety of natural records. Outcomes of the FM approach showing this behavior are presented along with a brief review of basic concepts on nonlinear time series analysis.

* Este artículo es un resumen de la tesis doctoral [Obregón, 1998] presentada en la University of California at Davis.

** Ingeniero Civil, Universidad Francisco de Paula Santander, Magíster en Ingeniería Civil con énfasis en Ingeniería de Recursos Hídricos, Universidad de los Andes, $\mathrm{PhD}$ en Hidrología, University of California at Davis. Profesor Asociado, Departamento de Ingeniería Civil, Pontificia Universidad Javeriana.

*** Matemático e Ingeniero Civil, Universidad de los Andes, MSc. en Investigación de Operaciones y en Ingeniería Civil, Massachusetts Institute of Technology, $\mathrm{PhD}$ en Hidrología, Massachusetts Institute of Technology. Associate Professor, Department of Land, Air and Water Resources, University of California at Davis. 


\section{Introduction}

In many fields of science one measures quantities that fluctuate in time or in space with no discernible patterns. Examples include magnetic and electric fields in plasmas, scalar quantities in fully-developed turbulence, weather and climatological data, variation of biological population, and stock prices. [Rowlands and Sprott, 1992] A common problem in the study of the irregular and apparently random motion of physical systems is to determine whether the system evolution is governed by some stochastic process (associated with a large number of active degrees of freedom) or if it may be interpreted in terms of the action of a few excited modes which have chaotic behavior. If a small number of excited modes dominates the dynamics then an approach based on the concept of deterministic chaos may be appropriate. In this case the motion may be described in terms of a system of a few ordinary differential equations, and systems with an apparently very complex behavior can be greatly simplified in terms of their physical description. [Provenzale et al., 1991] This has led to the hope that such simple systems can model the real world.

In this light, the detection of chaos as a possible mechanism underlying natural time series has attracted considerable attention. [Packard et al., 1980; Takens, 1981; Grassberger and Procaccia, 1983; Casdagli et al., 1991; Abarbanel et al., 1993] This has resulted in a battery of tests aimed at discriminating between chaotic (low-dimensional) and "random" (high-dimensional) behaviors which are applied in this work to study the dynamical properties of some generated measures obtained by the FM methodology.

\section{A Review of Nonlinear Time Series Analysis}

The recent interest in nonlinear dynamics, fractals, and chaotic systems has provided new insights into the working of many physical systems as well as a rapidly growing set of interpretative tools for nonlinear time series analysis. An assumption in such analysis is that a dynamical system in the form of a set of differential equations or discrete time maps in terms of a set of physical variables is responsible for the observed time series. Methods for recovering the dynamics of the system in terms of this single observable have been advanced. [Takens, 1981; Ruelle, 1994; Abarbanel et al., 1993; Casdagli et al. 1991] The recovered dynamics may be used to estimate invariants of the dynamics, as well as to make short term forecasts. If the reconstructed dynamics of the system is high dimensional, the time series of a single observed variable will be stochastic for all practical purposes, since a very large number of variables or coordinates are needed to model system evolution. 
On the other hand, if only few factors or variables determine the fate of that variable, describing its dynamics from a finite time series may be possible.

The correlation dimension [Grassberger and Procaccia, 1983] has been studied as a measure of complexity of a dynamical system and for distinguishing between low order, deterministic chaos and a random process. A measure related to the correlation dimension can be also be obtained using the nearest neighbor (NN) method firstly introduced by Pettis et al. [1979] and reproduced in various forms by other researchers. A geometrical method for dimension estimation has been devised by Abarbanel [1992]. This method uses nearest neighbors for each observation to check whether the trajectories of the underlying dynamics have been properly unfolded at a given embedding dimension. Among other methods to find the optimal embedding dimension is the "true vector field" approach of Kaplan and Glass [1992].

In order to review the concept of State Space Reconstruction from a scalar time series consider that the system of interest is characterized by a d-dimensional state space z. For an autonomous system the associated dynamics may be represented as:

$$
\frac{\mathrm{d} \mathbf{z}}{\mathrm{dt}}=\mathrm{F}(\mathbf{z}(\mathrm{t}))
$$

Now, consider a univariate time series $c_{l}, c_{2}, c_{t} \ldots$ for one of the $d$ state variables, c, generated by such a system, with sampling rate $\partial \mathrm{t}$. The system can be written as a higher order differential equation in terms of a single state variable $\mathrm{c}$,

$$
\mathrm{c}^{\mathrm{d}}=\mathrm{f}\left(\mathrm{c}, \dot{\mathrm{c}}, \ldots \ldots, \mathrm{c}^{\mathrm{d}-1}\right)
$$

Packard et al. [1980] and Takens [1981] introduced the notion of state space reconstruction from an observed scalar time series. A central idea here is the notion of phase space, i.e. the space defined by $\mathrm{c}(\mathrm{t})$, and its derivatives $\mathrm{c}(\mathrm{t}), \dot{\mathrm{c}}(\mathrm{t}), \ldots, \mathrm{c}^{\mathrm{d}}(\mathrm{t})$. The phase space in the univariate variable $c$, is a surrogate for the state space defined by $\mathbf{z}$. The derivatives $\dot{\mathrm{c}}(\mathrm{t}), \ldots, \mathrm{c}^{\mathrm{d}}(\mathrm{t})$, could be estimated numerically using a finite difference approximation to the time series $\mathrm{c}(\mathrm{t})$. However, such estimates induce an additional approximation error that grows with $d$. Noting that the available time series is sampled at discrete times $0, \partial \mathrm{t}, 2 \partial \mathrm{t}, \ldots, \mathrm{k} \partial \mathrm{t}$, one can define a pseudo-phase space using delay coordinates, i.e. by defining a delay vector $\mathrm{c}_{\mathrm{t}}=(\mathrm{c}(\mathrm{t}), \mathrm{c}(\mathrm{t}-\tau), \ldots, \mathrm{c}(\mathrm{t}-(\mathrm{m}-1) \tau))$, where $\tau$ is an appropriately chosen delay time, which is an integer multiple of $\partial \mathrm{t}$, and $m$ is an integer embedding dimension. 
If the solution to the equations lies on an attractor (i.e. a set of points, manifold or object in phase space that trajectories converge to after transients die out) of dimension $d_{\mathrm{A}}<d$, then choosing the integer $m>2 d_{\mathrm{A}}$ is a sufficient condition for unfolding the attractor from the scalar time series $\mathrm{c}(\mathrm{t}), \mathrm{t}=1 \ldots \mathrm{n}$. Then subject to generic assumptions on $\mathrm{f}$ and $\partial \mathrm{t}$, the underlying dynamics for any lag $\tau$, and forecast period T, could be represented by a smooth (i.e. differentiable) map [Casdagli et al., 1991]:

$$
c(t+T)=f^{T}(c(t), c(t-\tau), c(t-2 \tau), \ldots, c(t-(m-1) \tau)) ; \quad f^{T}: R^{m} \rightarrow R e
$$

This equation provides a basis for reconstructing a state space of the underlying dynamics given a scalar time series, as well as for forecasting that scalar component; provided the map or "regression" function $\mathrm{f}^{\mathrm{T}}$ can be described for appropriate values of $\tau$ and $m$. On the other hand, given enough data and a proper choice of the delay $\tau$, a stationary stochastic process will be space filling for any $m$ dimensional phase space (or $m$ delay coordinates).

A variety of prescriptions for choosing an appropriate $\tau$ have been presented in the literature. In general, if $\tau$ is too small, the coordinates will not be independent enough, that is to say that not enough time will have evolved for the system to have explored enough of its state space to produce, in a practical numerical sense, new information about its structure. [Abarbanel, 1996] In the case when $\tau$ is selected too large and the dynamics are chaotic, all relevant information for phase space reconstruction is lost since neighboring trajectories diverge, and averaging in time and/or space is no longer useful. There seems to be agreement (e.g. [Grassberger et al., 1991]) that there is no optimal method for choosing $\tau$. The goal is to end up with a coordinate set that is independent such that each coordinate added to the reconstruction set provides new information. Holzfuss and Mayer-Kress [1986] suggest it using a value of delay time at which the autocorrelation function first crosses the zero line. Tsonis and Elsner [1988] used a delay time greater than the decorrelation time, which they defined as the time at which the correlation drops to 1/e. Graf and Elbert [1990] wrote that the delay time can be set equal to the smallest lag for which the autocorrelation function is zero or to the first local minimum if that is earlier than the zero point. Another choice for the delay time is the value that produces the first local minimum in the mutual information function, $I$. [Fraser and Swinney, 1986] This last method has the advantage that it considers all kinds of relations, and not only the linear ones as in the autocorrelation function. $I$ attempts to measure how dependent the values of $c_{v \tau}$ are on the values of $c_{t}$ as a function of the time lag $\tau$. 
In the next section a dynamical study of some measures obtained by using the FM methodology is presented.

\section{The Fractal-Multifractal (FM) Methodology}

Inspired by the paradigms set forward by chaos theory, a simple geometric model which tries to represent (positive and normalized) natural time (or space) series in a deterministic and compact way was introduced recently by Puente [1992]. This model is based on the classic concept of derived distributions and assumes that natural sets may be understood as projections off fractal functions, built transforming simple multifractal measures (of relevance in fully-developed turbulence) [Meneveau and Sreenivasan, 1991] via fractal interpolating functions which are fixed points of affine mappings over two dimensions. [Barnsley, 1988] Given a multifractal measure $D X$ and a fractal interpolating function $\mathrm{f}$, the procedure then entails finding a derived measure $D Y=f^{-1} \square D X$, a fractional integration (over the crossings of $f$ ) of the parent measure $D X$. For an easier mental picture the reader is referred to the famous caveman's tale by Plato (Republic), such that natural sets become "shadows" $(D Y)$ coming from a "good" outside the cave $(f)$ which has a possibly non-uniform "illumination" $(D X)$.

Figure 1 shows two examples of derived measures generated by the procedure, termed $D Y 1$ and $D Y 2$, found transforming the same binomial multifractal measure $D X$ (having equal length scales and found via a cascade that splits the mass by its $60 \%$ ) via two fractal interpolating functions $\mathrm{f}_{1}$ and $\mathrm{f}_{2}$ which interpolate the same set of three points (dark circles at beginning, middle and end of functions domain) and whose graphs have fractal dimensions of 1.263 and 1, respectively. Clearly, $D Y 1$ and $D Y 2$ possess different appearances and textures. As seen, usage of the fractal interpolating function with higher dimension, $\mathrm{f}_{1}$, results in an integration of the parent measure $D X$ which appears to destroy much of its multifractality. Function $\mathrm{f}_{2}$, on the other hand, yields a measure which inherits the multiple layers present in $D X$. In this light, the purpose of this work is to show that the deterministic measures $D Y 1$ and $D Y 2$ possess indeed very different dynamic properties.

The graphs of fractal interpolating functions, being fixed points of suitable affine mappings, may be obtained point by point via iterations. [Barnsley, 1988] For the functions depicted in Figure 1 such mappings are:

$$
\mathrm{w}_{1}\left(\begin{array}{l}
\mathrm{x} \\
\mathrm{y}
\end{array}\right)=\left(\begin{array}{cc}
1 / 2 & 0 \\
1 & \mathrm{~d}_{1}
\end{array}\right)\left(\begin{array}{l}
x \\
y
\end{array}\right)
$$


Figure 1. Construction of deterministic measures $D Y 1$ and $D Y 2$.
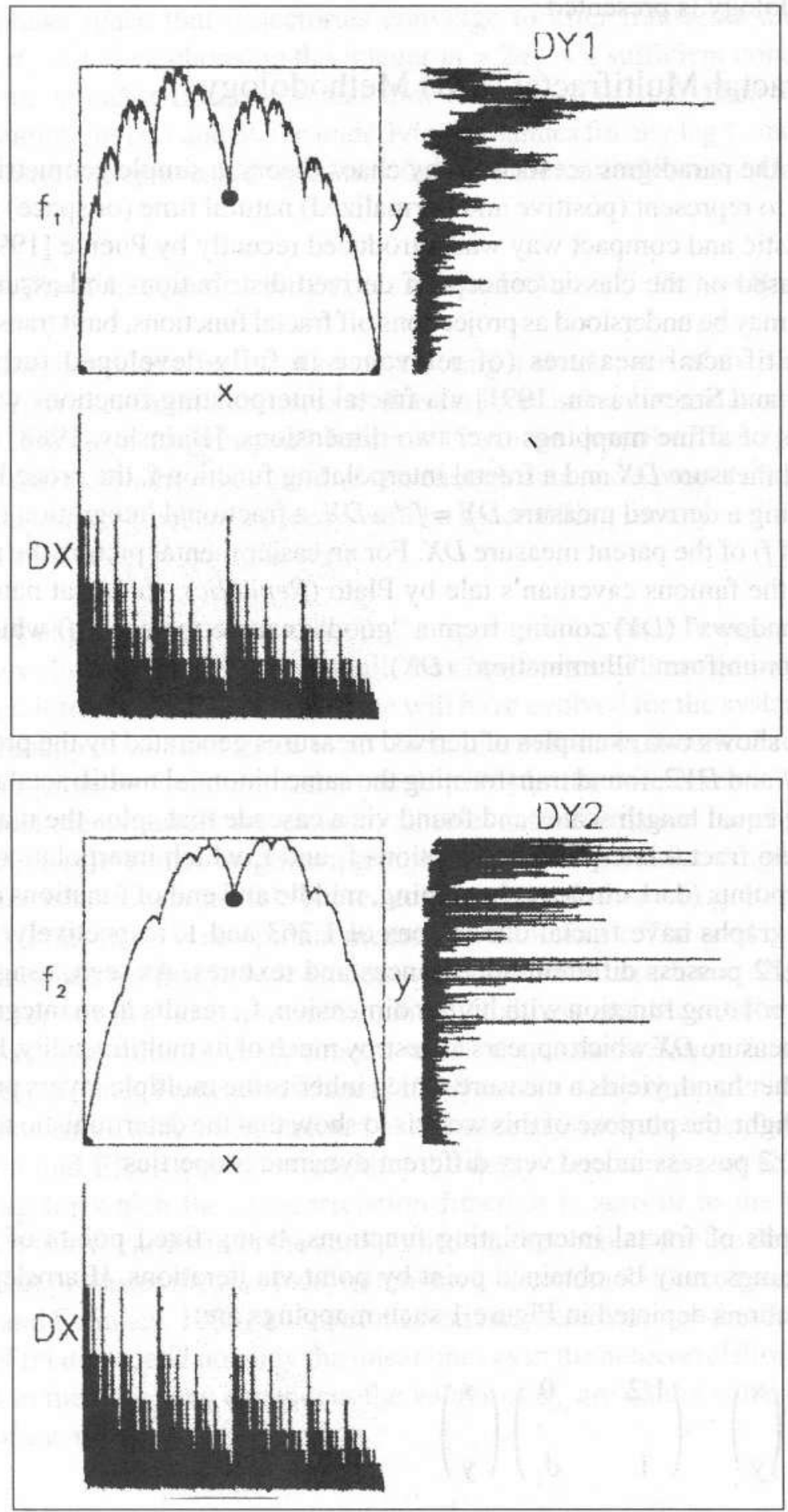


$$
w_{2}\left(\begin{array}{l}
x \\
y
\end{array}\right)=\left(\begin{array}{cc}
1 / 2 & 0 \\
1 & d_{2}
\end{array}\right)\left(\begin{array}{l}
x \\
y
\end{array}\right)+\left(\begin{array}{c}
1 / 2 \\
1
\end{array}\right)
$$

with $\mathrm{f}_{1}$ having $d_{1}=0.7$ and $d_{2}=0.5$, and $\mathrm{f}_{2}$ using $d_{1}=0.4$ and $d_{2}=0.5$. The stationary measures, over $x$ and $y$, correspond to histograms gathered during the iterations exercise [Elton, 1987], and yield the shown figures when $w_{1}$ and $w_{2}$ are chosen independently from time to time in proportions of $60 \%$ and $40 \%$, respectively. In order to fulfill series length requirements, both derived measures analyzed were sampled having $2^{16}$ values. To accomplish this task, the two affine mappings were iterated $800 \times 10^{6}$ times, starting at the point $(1 / 2,1)$, to define equally-spaced histograms of visited locations in $y$.

\section{Dynamical Properties of Derived Measures}

Figure 2 shows some of the most relevant statistics for both derived data sets. As seen, both sets may be termed "pink noises" as they yield power-law power spectra, $\mathrm{S}\left(\mathrm{f}^{-\beta}\right)$, over four cycles. As expected, DY2 (right) is inherently less correlated that DYI (left), as reflected in scaling exponents $\beta$ of 1.09 and 1.42 , respectively. The mass exponents $\tau$ for both records show that usage of a fractal interpolating function having a dimension larger than one, i.e. $\mathrm{f}_{1}$, indeed filter more readily the intermittency present in the parent multifractal measure $D X$. The entropy dimension $\left(D_{1}\right)$ values for both sets discriminate between them, with the less correlated series having a smaller entropy dimension. All these facts are reflected in Table 1 for time delays defined via first local minima $(\mathrm{flm})$ of both the autocorrelation $\rho(\tau)$ and the average mutual information function $I(\mathrm{t})$, and decay time to $\mathrm{e}^{-1} \mathrm{on} \rho(\tau)$ for both records.

Table 1. Time delays for phase-space reconstruction

\begin{tabular}{|c|r|r|}
\cline { 2 - 3 } \multicolumn{1}{c|}{} & DY1 & DY2 \\
\hline $\mathrm{I}(\mathrm{flm})$ & 133 & 20 \\
$\rho(\mathrm{flm})$ & 245 & 26 \\
$\rho\left(\mathrm{e}^{-1}\right)$ & 4860 & 472 \\
\hline
\end{tabular}


Figure 2. Sample statistics for deterministic measures DY1 (left) and DY2 (right). (i) Power spectrum $\mathrm{S}(\mathrm{f})$, (ii) mass exponents $(\tau(\mathrm{q})$ ), and (iii) percentage of false neighbors (\%FNN) for alternative delays $\tau$ as a function of embedding dimensions $m$.
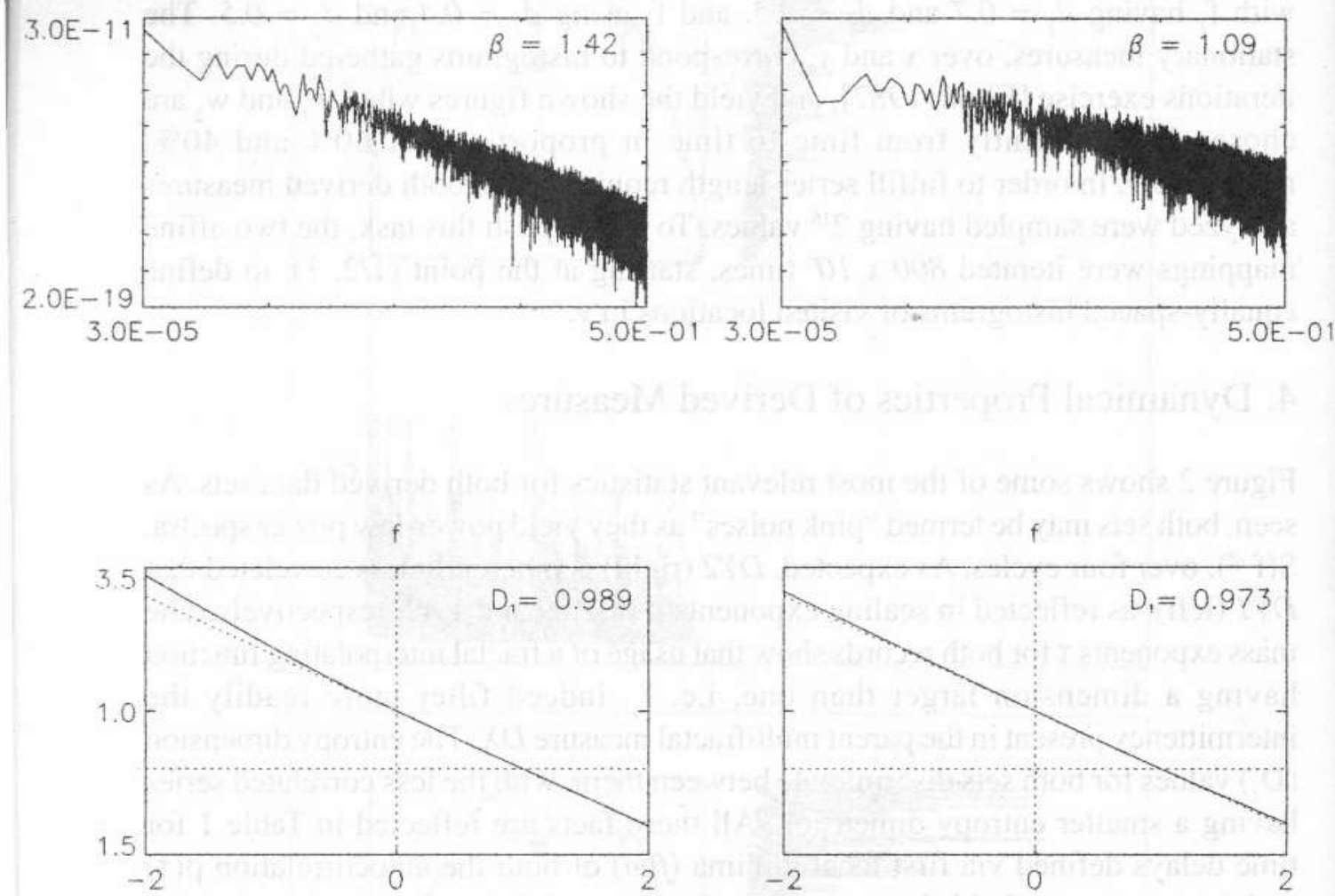

q
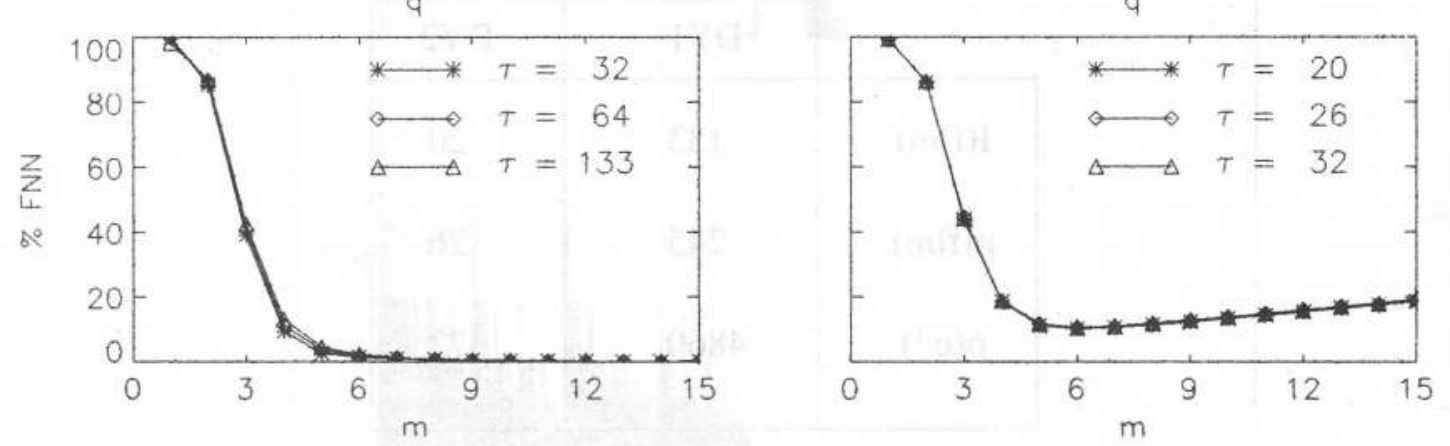
The bottom of Figure 2 summarizes the analysis of false near neighbors performed on both sets in an attempt to classify their inherent dynamics. Those figures include results for a variety of time delays which include the values based on the average mutual information function as reported in Table 1. As seen, the more correlated measure DY1 results in a stable embedding containing 5 or 6 coordinates, and hence could be termed low-dimensional. Analysis of maximum Lyapunov exponent for such attractor reveals indeed that $D Y 1$ may be classified as "chaotic." The more erratic DY2, on the other hand, does not show stabilization on the percentage of false near neighbors and hence may be termed dynamically highdimensional. These results are confirmed when correlation dimension analysis (not shown) is performed. The presence of a positive Lyapunov exponent for these attractors additionally reveals that $D Y 2$ would be classified by current methods as "stochastic."

The stabilized embedding dimension for $D Y 1$ and the lack of stabilization for $D Y 2$ appear to be related to the observation that stochastic processes with $1 / f^{\beta}$ power spectra yield stabilization in correlation dimension at a finite value of $D=2 /(\beta-1)$ [Osborne and Provenzale, 1989], i.e. $\beta$ of 1.42 gives $D=4.76$ and $\beta$ close to 1 gives $D$ which diverges. However, the data sets employed in this work are not random at all and usage of false near neighbors in addition to correlation dimensions suggest that the results are not anomalous but real for the generated records.

Notice that it is not trivial to have power-law power spectra on arbitrary derived measures nor for them to be even chaotic. In fact, generic (parent) deterministic multifractals or uniforms do not have power-law power spectrum scaling. And if fractal interpolating functions of increased dimensions are used, the derived measures tend to absolutely continuous distributions and limiting Gaussians [Puente, et al, 1996] whose dynamic properties yield only negative Lyapunov exponents. What the results suggest is the possibility of defining a wholistic language to approach the complexity of a vast class of natural sets via projections of deterministic functions whose parameters, when set in suitable ranges, yield derived measures whose dynamic behavior may be "chaotic" or "stochastic."

\section{Final remarks}

The vast range of derived measures that may be generated via the geometric procedure and the unexpected results presented herein hint that Plato's caveman metaphor may be the basis for developing a new vision to understand the intricacies of many data sets which otherwise would be classified as complex and "random". A detailed analysis (including an extensive statistical analysis) on the data sets 
that may be generated via the geometric procedure, the Fractal-Multifractal (FM), is also presented in [Obregón, 1998].

\section{References}

Abarbanel, H.D.I. Local False Nearest Neighbors and Dynamical Dimensions from Observed Chaotic Data, In: Physical Review, 47, 1992, 3057-3067.

Abarbanel, H.D.I., Brown, R., Sidorowich, J.J. and Tsimring, L.S. The Analysis of observed chaotic Data in physical Systems, In: Review of Modern Physic, 65, 1993, 1331.

Abarbanel, H.D.I. Analysis of Observed Chaotic Data. New York: Springer, 1996.

Barnsley, M.F. Fractals Everywhere. Academic Press, 1988.

Casdagli, M., Eubank, S., Farmer, J.D. and Gibson, J. State Space Reconstruction in the Presence of Noise, In: Physica D, 51, 1991, 52-98.

Elton, J. An Ergodic Theorem for Iterated Maps, In: Journal of Ergodic Theory and Dynamical Systems, 7, 1987, 481-488.

Fraser, A.M. and Swinney, H.L. Independent Coordinates for Strange Attractors from Mutual Information, In: Physical Review A, 33, 1986, 1134-1140.

Graf, K.E., Elbert, T. Dimensional Analysis of the Waking EEG, pp. 135-152. In: Basar, E. (Ed). Chaos in Brain Function. New York: Springer, 1990.

Grassberger, P., and Procaccia, I. Measuring the Strangeness of Strange Attractors. In: Physica $D, 9,1983,189-208$.

Grassberger, P., Schreiber, T. and Schaffrath, C. Non-Linear Time Sequence Analysis. University of Wuppertal, 1991.

Holzfuss, J. and Mayer-Kress, G. An Approach to Error-Estimation in the Application of Dimensions Algorithms. pp. 114-147. In: Mayer-Kress, G. Dimensions and Entropies in Chaotic Systems. New York: Springer, 1986.

Kaplan, D.T. and Glass, L. Direct Test for Determinism in a Time Series, In: Physical Review Letters, 68, 1992, 427-429.

Obregón, N. A New Geometric Approach to Study One-Dimensional Complex Data Sets. Doctoral Dissertation. University of California at Davis, 1998.

Osborne, A.R. Provenzale, A. Finite Correlation Dimension for stochastic Systems with power-law Spectra. In: Physica D, 35, 1989, 357.

Packard, N.H., Crutchfield, J.P., Farmer, J.D. and Shaw, R.S. Geometry from a Time Series. In: Physical Review Letters, 45, 1980, 712-716.

Pettis, K.W., Baley, T.A., Jain, A.K., and Dubes, R.C. An Intrinsic dimensionality Estimator from Near-neighbor Information. In: IEEE Transactions on Pattern Analysis and Machine Intelligence, PAMI-1(1), 1979, 25-37.

Plato, Republic. Oxford: Oxford University Press, 1993.

Provenzale, A., Osborne, A.R. and Soj, R. Convergence of the $\mathrm{K}_{2}$ Entropy for Random Noises with Power Law Spectra. In: Physica D, 47, 1991, 361-372.

Puente, C.E. Multinomial Multifractals, Fractal Interpolators, and the Gaussian Distribution. In: Physics Letters A, 161, 1992, 441-447.

Puente, C.E., López, M.M., Pinzón J.E., and Angulo, J.M. The Gaussian Distribution revisited. In: Advances in Applied Probability, 28, 1996, 500-524. 
Rowlands, G. and Sprott, J.C. Extraction of Dynamical Equations from Chaotic Data. In: Physica $D, 58,1992,251-259$.

Ruelle, D. ¿Where can One Hope to Profitably Apply the Ideas of Chaos?. In: Physics Today, 1994, 24-30.

Sreenivasan, K.R. Fractals and Multifractals in Fluid Turbulence. In: Annual Review of Fluid Mechanics, 23, 1991, 539-600.

Takens, F. Detecting strange Attractors in Turbulence. In: Rand, D. and Young, L.S. Dynamical Systems and Turbulence. Berlin: Springer, 1981.

Tsonis, A.A. and Elsner, J.B. The Weather Attractor over Very Short Timescales. In: Nature, $333,1988,545-547$. 